\title{
Propagation of solutions to the Fisher-KPP equation with slowly decaying initial data
}

\author{
Christopher Henderson *
}

September 25, 2018

\begin{abstract}
The Fisher-KPP equation is a model for population dynamics that has generated a huge amount of interest since its introduction in 1937. The speed with which a population spreads has been computed quite precisely when the initial data decays exponentially. More recently, though, the case when the initial data decays more slowly has been studied. Building on the results of Hamel and Roques '10, in this paper we improve their precision for a broader class of initial data and for a broader class of equations. In particular, our approach yields the explicit highest order term in the location of the level sets. In addition, we characterize the profile of the inhomogeneous problem for large times.
\end{abstract}

\section{Introduction}

In this article, we study the long time behavior of solutions to the Fisher-KPP equation

$$
\left\{\begin{array}{l}
u_{t}=u_{x x}+f(x, u), \quad t>0, x \in \mathbb{R}, \\
u(0, x)=u_{0}(x), \quad x \in \mathbb{R},
\end{array}\right.
$$

where $f$ is an $L$-periodic function in $x$, for some $L>0$, which vanishes when $u$ is 0 and 1 and where $u_{0}$ is a function taking values between 0 and 1 that decays slowly as $x$ tends to infinity. This equation comes from adding a diffusive term to the logistic equation, and it was originally introduced, with $f(x, u)=u(1-u)$, as model for population dynamics in the first half of the twentieth century [11,18. In this interpretation, $u$ stands for the local population density normalized by the carrying capacity, while $f$ accounts for the effect of an inhomogeneous environment on the growth of this population up to the carrying capacity.

Fisher and Kolmogorov, Petrovskii, and Piskunov showed that if the initial data is localized then the population would spread at a constant rate in the form of a traveling wave. This has been made more precise and succinct over time [5,6, 13, 15, 17, 22, 25, 26. In these works, the highest order terms in the spreading rate are obtained by studying the linearized problem. When $u_{0}$ is not localized, but decays exponentially, like $e^{-\lambda x}$, the behavior is similar. Here, the population converges to a traveling wave, the speed of which is determined by the order of the exponential decay and is given again by the linearized problem [26.

Recently, the case when $u_{0}$ decays slower than any exponential and $f$ is homogeneous was investigated by Hamel and Roques [16. They show that $u$ becomes asymptotically flat in time, which we will state precisely later. This implies that a traveling wave cannot give the limiting behavior of $u$. As a result, they investigate the location of the level sets of $u$ and show that, for each $m \in(0,1)$, the level sets satisfy

$$
\{x \in \mathbb{R}: u(t, x)=m\} \subset\left[u_{0}^{-1}\left(C_{m} e^{-f^{\prime}(0) t}\right), u_{0}^{-1}\left(c_{m} e^{-f^{\prime}(0) t}\right)\right],
$$

where $c_{m}<C_{m}$ are constants depending on $m$. These results hold for only a small class of initial data; for more general data, they show that level set is bounded above and below by $u_{0}^{-1}\left(\exp -\left(f^{\prime}(0) \pm \epsilon\right) t\right)$ for

\footnotetext{
*Department of Mathematics, Stanford University, Stanford, CA 94305, (chris@math.stanford.edu).
} 
any $\epsilon>0$. The main results of this paper are twofold: firstly, in the homogeneous setting, we explicitly characterize the constant in (1.2) for a broad class of the admissible initial data; secondly, we obtain equally precise results in the previously unstudied inhomogeneous setting. In fact, in the inhomogeneous setting, we show that $u$ grows at each point according to the solution to a generalized logistic equation. This provides an analogue to the asymptotic flatness result of Hamel and Roques in the inhomogeneous setting.

\section{Main results}

We now state our results more precisely. First we assume that there is a $\delta>0$ such that $f(x, u)$ is a positive, $C^{1, \delta}$ function with respect to $u$ that satisfies

$$
\left\{\begin{array}{l}
f(x, u) \leq f_{u}(x, 0) u \text { for all } u \in[0,1] \\
\frac{f(x, u)}{u} \text { is decreasing in } u, \text { and } \\
f_{u}(x, 0)>0 \text { and } \\
f_{u}(x, 1)<0
\end{array}\right.
$$

We note that some of the assumptions on the signs of $f$ and its derivatives can be relaxed, but we use them here to simplify the arguments.

We assume that $u_{0}$ is a positive function that decays to zero slower than any exponential. By this we mean that, for any $\epsilon \in(0, \infty)$

$$
\lim _{x \rightarrow \infty} u_{0}(x)=0, \quad \text { and } \quad \lim _{x \rightarrow \infty} u_{0}(x) e^{\epsilon x}=\infty .
$$

For instance, functions which decay algebraically as $x$ tends to infinity satisfy the conditions in (2.4). Though our technique can handle any functions of this type, the presentation is much simpler if we restrict to initial data, $u_{0}$, which are eventually monotonic and which decay slower than $e^{-\beta \sqrt{x}}$ for any $\beta$. Namely, we assume that there is a point $x_{0}>0$ such that

$$
\begin{aligned}
& u_{0}^{\prime}(x)<0 \text { for all } x \geq x_{0}, \quad \text { and } \\
& \lim _{x \rightarrow \infty} \frac{u_{0}^{\prime}(x)}{u_{0}(x) / \log \left(u_{0}(x)\right)}=0 .
\end{aligned}
$$

We will comment on the effect of this assumption later. In addition, since we are studying the effect of a slowly decaying tail at $\infty$, we additionally assume that $u_{0}$ is front-like. In other words, we assume that

$$
\liminf _{x \rightarrow-\infty} u_{0}(x)>0
$$

Again, we note that the positivity assumption on $u_{0}$ can be relaxed, but we use it here to simplify the presentation.

Classical results address the case when $u_{0} \sim e^{-\lambda x}$ [5, 6, 11, 18, 22, 26]. If $\lambda>1$ then $u$ converges to a traveling wave with speed 2 in the correct moving frame, while if $\lambda<1, u$ converges to a traveling wave with speed $\lambda+1 / \lambda$ in the moving frame. In short, this means that the level sets of $u_{0}$ move with a constant speed, which increases to infinity as $\lambda$ decreases to zero, and that any two level sets travel at the same speed and remain a bounded distance from one another for all time. Hamel and Roques point out in [16] that, using these as a family of sub-solutions, we can see immediately that the level sets of any solution with initial data like eq. (2.4) will spread to the right super linearly in time. We now calculate the speed of these level sets explicitly.

\subsection{The homogeneous case}

Our first result concerns the spreading of the level sets in the homogeneous setting. 
Theorem 2.1. Suppose that $u$ solves eq. (1.1) with $f$ depending only on $u$ and not on the space variable, $x$. Suppose further that $f$ satisfies condition (2.3) and that $u_{0}$ satisfies conditions (2.4) and (2.7). In addition, let $u_{0}$ satisfy conditions (2.5) and (2.6). Let $\varphi$ be the unique (up to translation) global in time solution to

$$
\varphi_{t}=f(\varphi) .
$$

Then, for each $m \in(0,1)$, define $T_{m} \in \mathbb{R}$ to be the unique time so that $\varphi\left(T_{m}\right)=m$. Then, for any $r>0$, we have

$$
\limsup _{T \rightarrow \infty} \sup _{x \geq u_{0}^{-1}\left(\varphi\left(T_{m}-T\right)\right)-r T} u(T, x) \leq m .
$$

and

$$
\liminf _{T \rightarrow \infty} \inf _{x \leq u_{0}^{-1}\left(\varphi\left(T_{m}-T\right)\right)+r T} u(T, x) \geq m .
$$

In order to make this result clearer, we first consider a specific example. If $f$ is the standard Fisher-KPP non-linearity, i.e. $f(u)=u(1-u)$, then

$$
\varphi(T)=\frac{e^{T}}{1+e^{T}}, \quad \text { implying that } \quad \varphi\left(T_{m}-T\right)=\frac{m}{1-m} e^{-T}+O\left(e^{-2 T}\right),
$$

where $O\left(e^{-2 T}\right)$ is bounded by a constant multiple of $e^{-2 T}$ for all $T$. For a general non-linearity, $f$, one can easily see (by linearization) that, up to leading order, $\varphi\left(T_{m}-T\right)=c_{m} e^{-f^{\prime}(0) T}$, where $c_{m}$ is a constant depending only on $f$ and $m$. In addition, defining the level set of $u$ as

$$
E_{m}(t) \stackrel{\text { def }}{=}\{x: u(t, x)=m\}
$$

if we look at, e.g., initial data such that $u_{0}(x)=x^{-\alpha}$ for large enough $x$, theorem 2.1 implies that

$$
E_{m}(t) \sim\left(\frac{1-m}{m} e^{t}\right)^{1 / \alpha}
$$

Notice that the non-linearity affects the constant $\frac{1-m}{m}$, as the linearized equation would yield only $1 / m$. The fact that the full non-linearity affects the highest order behavior is in contrast to the case when $u_{0}$ decays exponentially, where the linearized equation completely determines the highest order behavior.

An easy corollary of theorem 2.1 is the following.

Corollary 2.2. For any $\epsilon>0$, under the assumptions of theorem 2.1, there exists $T_{0}>0$ depending on all parameters such that if $T>T_{0}$ then

$$
E_{m}(T) \subset\left[u_{0}^{-1}\left(\varphi\left(T_{m+\epsilon}-T\right)\right), u_{0}^{-1}\left(\varphi\left(T_{m-\epsilon}-T\right)\right)\right] .
$$

Again, it is instructive to look at the example that we considered above. Corollary 2.2 implies that, for any $\epsilon$ and any $t$ large enough, we have

$$
E_{m}(t) \subset\left[\left(\frac{m}{1-m}-\epsilon\right)^{1 / \alpha} e^{t / \alpha},\left(\frac{m}{1-m}+\epsilon\right)^{1 / \alpha} e^{t / \alpha}\right] .
$$

Remark 2.3. We comment briefly on the assumption on the decay of the first derivative of $u_{0}$, eq. (2.6). In practice, this means that $u_{0}$ decays slower than $e^{-\sqrt{x}}$. We could remove this restriction, assuming only that $u_{0}$ satisfies eq. (2.4). In this case, we would obtain a linear in time error term in eqs. (2.8) and (2.9) and in corollary 2.9. In other words, there is a constant $r>0$ such that, for any $m \in(0,1)$ and $\epsilon>0$, we have

$$
E_{m}(T) \subset\left[u_{0}^{-1}\left(\varphi\left(T_{m+\epsilon}-T\right)\right)-r T, u_{0}^{-1}\left(\varphi\left(T_{m-\epsilon}-T\right)\right)+r T\right],
$$

for $T$ large enough. We omit proving this here, in the interest of presenting a single, clear argument and result. 


\subsection{The inhomogeneous case}

When the non-linearity, $f$, depends periodically on $x$, the level sets fail to capture the spreading behavior. Intuitively this is because the function $u_{0}$ is very flat at infinity, so it will grow as if it were a solution to the periodic, inhomogeneous Fisher-KPP equation. In particular, $u$ will oscillate in space enough that the width of the level sets will grow to infinity as $t$ tends to infinity. In other words, the distance between the rightmost and leftmost points of the level set $E_{m}(t)$ tends to infinity.

To circumvent this, we look instead at the locations of the intervals of length $L$, the length of the period of $f$, of a given mean oscillation. In other words, we seek to describe the location of the average-level

$$
\bar{E}_{m}(t) \stackrel{\text { def }}{=}\left\{x \in \mathbb{R}: f_{x}^{x+L} u(t, y) d y=m\right\},
$$

where we define, for any interval $[a, b]$ and any function $\gamma(x)$,

$$
f_{a}^{b} \gamma(x) d x \stackrel{\text { def }}{=} \frac{1}{|b-a|} \int_{a}^{b} \gamma(x) d x .
$$

Using $\bar{E}_{m}(t)$, we find similar results to theorem 2.1 in the inhomogeneous setting.

As we alluded to above, instead of the logistic equation, the behavior of $u$ on each interval of length $L$ is captured by the global in time solution to

$$
\left\{\begin{array}{l}
\varphi_{t}=\varphi_{x x}+f(x, \varphi), \quad \text { for all }(t, x) \in \mathbb{R} \times \mathbb{T} \\
f \varphi(0, x) d x=\frac{1}{2},
\end{array}\right.
$$

where $\mathbb{T}=[0, L]$ with the ends identified. In other words, $\varphi$ is an $L$-periodic solution to the equation above. We briefly note that, although the techniques to show the well-posedness of eq. (2.11) are similar to those used in the theory of well-posedness for the Fisher-KPP equation, see e.g. [2], we are unable to find a treatment of it in the literature. As such, we dedicate section 6 to proving the existence and uniqueness of the solution to this problem.

We define one last quantity before we state our results. For each $m \in(0,1)$ and each $T>0$, we may solve the terminal value problem

$$
\left\{\begin{array}{l}
\varphi_{t}^{T, m}=\varphi_{x x}^{T, m}+f\left(x, \varphi^{T, m}\right), \quad \text { on }[0, T] \times[0, L], \\
\frac{1}{L} \int_{0}^{L} \varphi^{T, m}(T, x) d x=m, \\
\varphi^{T, m}(0) \equiv B(m, T)
\end{array}\right.
$$

with periodic boundary conditions and where $B(m, T)$ is an unknown number depending only on $f$, $m$, and $T$. It is simple to check that the system eq. (2.12) is well defined and is continuous in $m$ and $T$. It is also easy to check that $B(m, T)$ is increasing in $m$ and decreasing in $T$.

We now formulate our results precisely.

Theorem 2.4. Suppose that $u$ solves eq. (1.1) and that $f$ is an L-periodic function satisfying the conditions in eq. (2.3). Suppose $u_{0}$ satisfies the conditions in eqs. (2.4) to (2.7). For any $r>0$, we have that

$$
\limsup _{T \rightarrow \infty} \sup _{x \geq u_{0}^{-1}(B(m, T))-r T} f_{x}^{x+L} u(T, y) d y \leq m,
$$

and

$$
\liminf _{T \rightarrow \infty} \inf _{x \leq u_{0}^{-1}} f_{B(m, T))+r T}^{x+L} u(T, y) d y \geq m,
$$

where $B(m, T)$ is defined in (2.12). 
This result gives an analogous result to the theorem 2.1. Namely, that to find an interval $[n L, n L+L]$ such that $u(t, x)$ take the value $m$ on average, one has to choose $n$ such that $u_{0}(x)$ roughly looks like $B(m, T)$ for $x \in[n L, n L+L]$.

In theorem 2.4, the $B(m, T)$ term is a bit mysterious. In the homogeneous case, we characterize the location of the level sets in terms of the global-in-time solution to the logistic equation, an ordinary differential equation. The following proposition does the same here, making rigorous the ODE-like behavior of $u$ by characterizing $B(m, T)$ in terms of solution to a global-in-time analogue to the logistic equation.

Proposition 2.5. Let the conditions of theorem 2.4 be satisfied. Let $\psi_{0}$ and $f_{0}$ be the unique positive solutions to the eigenvalue problem

$$
\left\{\begin{array}{l}
\left(\psi_{0}\right)_{x x}+f_{u}(x, 0) \psi_{0}=f_{0} \psi_{0}, \quad x \in \mathbb{T} \\
\int \psi_{0}^{2} d x=1
\end{array}\right.
$$

Let $T_{m}$ be the unique time that $f \varphi\left(T_{m}\right) d x=m$. Then

$$
\lim _{T \rightarrow \infty} \frac{B(m, T) \int \psi_{0}(x) d x}{\int \varphi\left(T_{m}-T, x\right) \psi_{0}(x) d x}=1 .
$$

Again we may obtain an easy corollary regarding the movement of the average-level sets $\bar{E}_{m}(t)$.

Corollary 2.6. Let the hypotheses of proposition 2.5 be satisfied. For any $\epsilon>0$, and any $m \in(0,1)$ there is a time $T_{0}$, depending on all parameters, such that if $T \geq T_{0}$, then

$$
\bar{E}_{m}(t) \subset\left[u_{0}^{-1}\left(\varphi\left(T_{m+\epsilon}-T\right)\right), u_{0}^{-1}\left(\varphi\left(T_{m-\epsilon}-T\right)\right)\right] .
$$

As a part of the proof, we will show that there is a positive constant $\alpha>0$ such that, for very large negative times, $\varphi(t, x) \sim \alpha e^{f_{0} t} \psi_{0}(x)$. Hence proposition 2.5 and corollary 2.6 imply that there is a constant $c_{m}$, depending only on $m$ and $f$, such that

$$
\bar{E}_{m}(t) \sim u_{0}^{-1}\left(c_{m} e^{-f_{0} t}\right)
$$

holds for large times $t$. The constant $c_{m}$ is, in principle, computable given a specific non-linearity $f$ by solving the global in time periodic problem.

Finally, we prove a result which is analogous to the asymptotic flatness result of [16]. This final result shows that $u$ grows exactly as the global in time solution of the periodic problem.

Theorem 2.7. Let the conditions of theorem 2.4 be satisfied. Let $\psi_{0}$ be as in proposition 2.5. Define

$$
S_{n}=\left(\int \psi_{0}(x) d x\right)^{2} u_{0}(n L) .
$$

Then

$$
\lim _{T \rightarrow \infty} \max _{n \in \mathbb{Z}}\left\|u(T, \cdot)-\varphi\left(T^{S_{n}}+T, \cdot\right)\right\|_{L^{\infty}([n L, n L+L])}=0
$$

\section{Related work}

We will now briefly describe related work. As we have mentioned above, the most closely related work is that of Hamel and Roques [16. In this novel article, the authors present three main results regarding the solutions to the homogeneous Fisher-KPP equation (i.e. (1.1) with $f$ depending only on $u$ ). First, they show that $\left\|u_{x} / u\right\|_{L^{\infty}(\mathbb{R})} \rightarrow 0$ as $t$ tends to infinity. Further, they show that if the initial data decays slower than $x^{-\alpha_{f}}$, for some $\alpha_{f}$ depending only on the smoothness of $f$, there are constants $C_{m}$ and $C$ such that, for $t$ large enough,

$$
E_{m}(t) \subset\left[u_{0}^{-1}\left(C_{m} \exp -f^{\prime}(0) t\right), u_{0}^{-1}\left(C m \exp -f^{\prime}(0) t\right)\right] .
$$


We note that $\alpha_{f}=2$ when $f(u)=u(1-u)$. Lastly, for more general initial data, they obtain the exponential order. Precisely, they show that, for any $\epsilon$,

$$
E_{m}(t) \subset\left[u_{0}^{-1}\left(\exp -\left(f^{\prime}(0)-\epsilon\right) t\right), u_{0}^{-1}\left(\exp -\left(f^{\prime}(0)+\epsilon\right) t\right)\right],
$$

holds for any $t$ large enough.

Hamel and Roques obtain their bounds on $E_{m}(t)$ by carefully building sub- and super-solutions using the function, $u_{0}(x) e^{f^{\prime}(0) t}$, and using the fact that we may bound $f$ below by a second order Taylor approximation at zero. Our method involves quite different sub- and super-solutions, and the difference in precision between the constant in their bounds and our bounds, in eq. (2.10), can be attributed to the fact that our sub- and super-solutions feel the full affect of the non-linearity, $f$.

Beyond the work of Hamel and Roques, there has been recent interest in super-linear in time propagation phenomena, or accelerating fronts. In a series of articles [7 9], Cabré, Coulon, and Roquejoffre investigated the phenomenon of exponential spreading of level sets of solutions to the Fisher-KPP with the fractional Laplacian. In both cases, the mechanism is the same: when the data is spread out, $u(\cdot, x)$ evolves according to the logistic equation for every $x$. In this model, the fat tails of the fractional Laplacian are responsible for spreading $u$ out in finite time. We note that our techniques can be adapted to yield analogous results when $u_{0}$ decays slower than the critical cases investigated by Cabré, Coulon, and Roquejoffre. These accelerating fronts have also been observed in biological models with a non-local dispersal operator with fat tails [12 19 23] and in a kinetic reaction-transport equation [4. Finally, they have been conjectured to occur in a reactiondiffusion-mutation model with variable motility [3].

\section{Plan of the paper}

The results of theorem 2.1 are a special case of theorem 2.4, and, hence, we only prove theorem 2.4. We do so in section 3 by creating sub- and super-solutions using the heat equation and eq. (2.12), with carefully chosen initial data for both. Then we prove proposition 2.5 in section 4 through an eigenfunction decomposition. This characterizes the location of the level sets in terms of the global in time problem eq. 2.11). In section 5 , we prove theorem 2.7, showing that at large times $u$ locally looks like the global in time solution to eq. (2.11). Finally, we show that eq. (2.11) admits a unique global in time solution in section 6 .

\section{Notation}

In order to make the discussion clearer, we adopt the following notation. The constant, $C$, denotes an arbitrary constant independent of time which may change line-by-line. In addition, we occasionally use $o(1)$ to mean a constant that tends to zero along with some quantity which we will specify. We will make clear, in each usage, the dependencies of $C$ and $o(1)$.

\section{$3 \quad$ Spreading of the level-average sets}

In this section, we prove theorem 2.4 by creating sub- and super-solutions by decomposing $u$ into a product of purely diffusive and purely reactive terms. We utilize the Feynman-Kac formula along with our condition on the oscillations, eq. (2.6), in order to control the diffusive term.

In addition, we state a lemma which will allow us to translate the simple condition on the decay of $u_{0}$, eq. (2.6), to a more useful condition on the oscillations of $u_{0}$ very far to the right.

Lemma 3.1. Suppose that $u_{0} \in C^{1}$ is strictly decreasing and satisfies

$$
\lim _{x \rightarrow \infty} \frac{\left(u_{0}\right)_{x}(x)}{u_{0}(x) / \log \left(u_{0}(x)\right)}=0 .
$$

Let $\lambda_{t}$ be some quantity for which there exist constants $c_{1}, c_{2}>0$ such that $\lambda_{t} \leq c_{1} e^{-c_{2} t}$. Then, for any choice of $c_{3}$, we have that

$$
\lim _{t \rightarrow \infty} \frac{u_{0}\left(u_{0}^{-1}\left(\lambda_{t}\right) \pm c_{3} t\right)}{\lambda_{t}}=1
$$


The limit may be taken uniformly for any $c_{1}$ which is bounded above.

We will use this with the choice $\lambda_{t}=B(m, t)$. To this end, we need the following lemma that guarantees the exponential decay of $B(m, t)$ in time, which will be useful in its own right.

Lemma 3.2. Let $B(m, T)$ be defined as in theorem 2.4. Let $f_{0}$ be the largest eigenvalue of $\Delta+f_{u}(x, 0)$. Then $f_{0}>0$ and there exists positive constants $C$, independent of $m$, and $C_{m}$, depending on $m$, such that

$$
\frac{m}{C} e^{-f_{0} T} \leq B(m, T) \leq C_{m} e^{-f_{0} T} .
$$

Moreover, there exists $\delta_{0}>0$ such that if $m \leq \delta_{0}$, then

$$
B(m, T) \leq C m e^{-f_{0} T} .
$$

We prove these lemmas in section 3.3 .

\subsection{An upper bound}

To begin, we define

$$
x_{m}(T)=u_{0}^{-1}(B(m, T)),
$$

and we define a super-solution as $\bar{v}=\varphi^{T, m} \bar{w}$ where $\varphi^{T, m}$ satisfies eq. (2.12) and $\bar{w}$ satisfies

$$
\left\{\begin{array}{l}
\bar{w}_{t}=\bar{w}_{x x}+2\left(\frac{\varphi_{x}^{T, m}}{\varphi^{T, m}}\right) \bar{w}_{x}, \\
\bar{w}(0, x)=\max \left\{\frac{u_{0}(x)}{B(m, T)}, 1\right\} .
\end{array}\right.
$$

Indeed, we notice that $\bar{v}(0, x) \geq u_{0}(x)$ for every $x$ and that

$$
\begin{aligned}
\bar{v}_{t}-\bar{v}_{x x}-f(x, \bar{v})= & \varphi_{t}^{T, m} \bar{w}+\varphi^{T, m} \bar{w}_{t}-\varphi_{x x}^{T, m} \bar{w}-2 \varphi_{x}^{T, m} \bar{w}_{x}-\varphi^{T, m} \bar{w}_{x x}-f\left(x, \varphi^{T, m} \bar{w}\right) \\
= & {\left[\varphi_{x x}^{T, m}+f\left(x, \varphi^{T, m}\right)\right] \bar{w}+\varphi^{T, m}\left[\bar{w}_{x x}+2 \frac{\varphi_{x}^{T, m}}{\varphi^{T, m}} \bar{w}_{x}\right]-\varphi_{x x}^{T, m} \bar{w}-2 \varphi_{x}^{T, m} \bar{w}_{x} } \\
& -\varphi^{T, m} \bar{w}_{x x}-f\left(x, \varphi^{T, m} \bar{w}\right) \\
= & \bar{w} \varphi^{T, m}\left[\frac{f\left(x, \varphi^{T, m}\right)}{\varphi^{T, m}}-\frac{f\left(x, \varphi^{T, m} \bar{w}\right)}{\bar{w} \varphi^{T, m}}\right] \geq 0 .
\end{aligned}
$$

The inequality in the last line follows from the fact that $\bar{w} \geq 1$, by the maximum principle, and from the fact that $f(x, s) / s$ is decreasing in $s$ by eq. (2.3).

As a result of the above decomposition, we need only show that $\bar{w}$ does not deviate much from 1 near $x_{m}(T)$. To this end, we will use the Feynman-Kac representation of $\bar{w}$, see e.g. 1]. First, it follows from the standard parabolic regularity theory that

$$
\left\|\varphi_{x}^{T, m} / \varphi^{T, m}\right\|_{\infty} \leq C_{0}
$$

where $C_{0}$ is a constant independent of $t, T$, and $m$, see e.g. 20,21. We define a random process $X_{t}$ as the solution to

$$
\left\{\begin{array}{l}
d X_{t}=2 \frac{\varphi_{x}^{T, m}\left(T-t, X_{t}\right)}{\varphi^{T, m}\left(T-t, X_{t}\right)} d t+\sqrt{2} d B_{t}, \\
X_{0}=x,
\end{array}\right.
$$

where $B_{t}$ is a standard Brownian motion. We may then represent $w$ as

$$
\bar{w}(t, x)=\mathbb{E}^{x}\left[\bar{w}\left(0, X_{t}\right)\right] .
$$

Fix $r_{1}>0$, to be determined later, and any $r_{2}$. For $x \geq x_{m}(T)-r_{2} T$, we estimate this at time $T$ as

$$
\bar{w}(T, x)=\mathbb{E}^{x}\left[\bar{w}\left(0, X_{T}\right) \mathbb{1}_{\left\{X_{T} \leq x-r_{1} T\right\}}\right]+\mathbb{E}^{x}\left[\left(\bar{w}\left(0, X_{T}\right)-1\right) \mathbb{1}_{\left\{X_{t} \geq x-r_{1} T\right\}}\right]+\mathbb{E}^{x}\left[\mathbb{1}_{\left\{X_{t} \geq x-r_{1} t\right\}}\right] .
$$


First, we may use lemma 3.2 to find a constant $C$ with which we may bound $\|\bar{w}(0)\|_{\infty} \leq C e^{f_{0} T}$. Then, if $r>2 C_{0}+2 \sqrt{f_{0}}$, with $C_{0}$ as in eq. (3.20), the first term in eq. $(3.22)$ may be bounded as

$$
\begin{aligned}
\mathbb{E}^{x}\left[\bar{w}\left(0, X_{T}\right) \mathbb{1}_{\left\{X_{T} \leq x-r T\right\}}\right] & \leq C e^{f_{0} T} \mathbb{P}\left\{X_{T} \leq x-r T\right\} \leq C e^{f_{0} T} \mathbb{P}\left\{\sqrt{2} B_{T}-2 C_{0} T \leq-r T\right\} \\
& \leq C e^{f_{0} T} \mathbb{P}\left\{\sqrt{2} B_{T} \leq-2 \sqrt{f_{0}} T\right\} \leq \frac{C}{\sqrt{T}} .
\end{aligned}
$$

The last inequality follows from a standard estimate on the tail of a Gaussian. Thus, the first term tends to zero as $T$ tends to infinity. The third term in eq. (3.22) can be shown to converge to 1 in the exact same manner as above. Notice that these limits did not depend on $m$.

Hence, we need only show that the second term converges to zero. To this end we bound the second term as

$$
\begin{aligned}
\mathbb{E}^{x}\left[\left(\bar{w}\left(0, X_{T}\right)-1\right) \mathbb{1}_{\left\{X_{t} \geq x-r_{1} T\right\}}\right] & \leq \mathbb{E}^{x}\left[\frac{u_{0}\left(x-r_{1} T\right)-u_{0}\left(x_{m}(T)\right)}{B(m, T)}\right] \\
& \leq \mathbb{E}^{x}\left[\frac{u_{0}\left(x_{m}(T)-\left(r_{1}+r_{2}\right) T\right)-u_{0}\left(x_{m}(T)\right)}{B(m, T)}\right] .
\end{aligned}
$$

Using the definition of $x_{m}(T)$, eq. (3.18), this tends to zero as a consequence of lemma 3.1 and lemma 3.2 . We notice that this limit can be taken independent of $m$ if $m$ is bounded away from 1 as a consequence of the tracking the dependence on $m$ through lemma 3.1 and lemma 3.2 .

Hence we obtain that if $x \geq x_{m}(T)-r_{2} T$, then $\bar{w}(T, x) \leq 1+o(1)$, where $o(1)$ tends to zero as $T$ tends to infinity. This yields

$$
u(T, y) \leq \varphi^{T, m}(T, y) \bar{w}(T, y) \leq \varphi^{T, m}(T, y)[1+o(1)] .
$$

By construction, $f \varphi^{T, m}(T, y) d y=m$. Hence, averaging over $[x, x+L]$ gives us the upper bound eq. (2.13).

We note that, to take the limits above, we used only estimates on the Gaussian and lemma 3.1 and lemma 3.2. Hence, these limits can be taken independent of $m$ as long as $m$ is uniformly bounded away from 0 and 1 .

\section{$3.2 \quad$ A lower bound}

The argument here is similar to the argument in section 3.1, above. As before we fix $T>0$ and $m \in(0,1)$, define $x_{m}(T)$ as in eq. (3.18), and we $\underline{v}(t, x)=\varphi^{T, m}(t, x) \underline{w}(t, x)$ where $\varphi^{T, m}(t, x)$ is defined in eq. (2.12). Here, we instead define $\underline{w}$ as the solution to

$$
\left\{\begin{array}{l}
\underline{w}_{t}=\underline{w}_{x x}+2 \frac{\varphi_{x}^{T, m}}{\varphi^{T, m}} \underline{w}_{x}, \\
\underline{w}(0, x)=\min \left\{\frac{u_{0}(x)}{B(m, T)}, 1\right\} .
\end{array}\right.
$$

Notice that $\underline{v}(0, x) \leq u_{0}(x)$ for all $x$. The computation above, eq. (3.19), shows that

$$
\underline{v}_{t}-\underline{v}_{x x}-f(x, \underline{v})=\underline{w} \varphi^{T, m}\left[\frac{f\left(x, \varphi^{T, m}\right)}{\varphi^{T, m}}-\frac{f\left(x, \varphi^{T, m} \underline{w}\right)}{\underline{w} \varphi^{T, m}}\right] \leq 0,
$$

where the last inequality follows since $\underline{w} \leq 1$, as a consequence of the maximum principle, and since $f(x, s) / s$ is decreasing for $s \in[0,1]$.

Again, defining $X_{t}$ by eq. (3.21), we obtain that

$$
\underline{w}(t, x)=\mathbb{E}^{x}\left[\underline{w}\left(0, X_{t}\right)\right] .
$$

Fix $r_{1}>0$, to be determined below, and any $r_{2}$. For any $x \leq x_{m}(T)+r_{2} T$, we decompose $w(T, x)$ as

$$
\underline{w}(T, x)=\mathbb{E}^{x}\left[\underline{w}\left(0, X_{T}\right) \mathbb{1}_{\left\{X_{T} \geq x+r_{1} T\right\}}\right]+\mathbb{E}^{x}\left[\left(\underline{w}\left(0, X_{T}\right)-1\right) \mathbb{1}_{\left\{X_{t} \leq x+r_{1} T\right\}}\right]+\mathbb{E}^{x}\left[\mathbb{1}_{\left\{X_{t} \leq x+r_{1} T\right\}}\right] .
$$


For $x \leq x_{m}(T)+r_{2} T$, we may again choose $r_{1}$ large enough that the first term on the right hand side tends to zero as $T$ tends to infinity and that the last term on the right tends to one as $T$ tends to infinity. In addition, lemma 3.1 and lemma 3.2 imply that the second term on the right tends to zero as $T$ tends to infinity. Hence, we obtain, for any $x \leq x_{m}(T)+r_{2} T$,

$$
u(T, x) \geq \varphi^{T, m}(T, x) \underline{w}(T, x) \geq \varphi^{T, m}(T, x)(1-o(1)) .
$$

Using that, by construction, $f \varphi^{T, m}(T, y) d y=m$, integrating over $[x-L, x]$ yields the lower bound in eq. (2.14) for any $x \leq x_{m}(T)+r_{2} T$. Again, we note that the limit in eq. (3.24) may be taken uniformly for $m$ bounded away from 1 .

\subsection{Controlling the oscillations}

In this section we prove lemma 3.1 and lemma 3.2. We first prove lemma 3.1, by using an ODE argument to compare $u_{0}$ to a sequence of equations which decay at the critical order but with increasingly slower rates.

Proof of lemma 3.1. We will prove this lemma for $u_{0}^{-1}\left(\lambda_{t}\right)-c_{3} t$, though the proof is exactly analogous in the case of $u_{0}^{-1}\left(\lambda_{t}\right)+c_{3} t$. Fix $\epsilon>0$, and define $h_{\epsilon}(x)=e^{-\sqrt{\epsilon x}}$. Notice that

$$
h^{\prime}=-\frac{\epsilon}{2} \frac{h}{\log (h)} \text {. }
$$

Define

$$
x_{0}=u_{0}^{-1}\left(\lambda_{t}\right) \quad \text { and } \quad y_{0}=h^{-1}\left(\lambda_{t}\right) .
$$

We may then shift $h$ to obtain $g(x)=h\left(x-x_{0}+y_{0}\right)$. Notice that $g\left(x_{0}\right)=u_{0}\left(x_{0}\right)$. The condition on the decay of $\lambda_{t}$ and the fact that $u_{0}$ decays slower than any exponential implies that $u_{0}^{-1}\left(\lambda_{t}\right)-c_{3} t$ tends to infinity. Hence we may choose $t$ large enough, uniformly in $c_{1}$ if $c_{1}$ is bounded above, such that if $x \geq x_{0}-c_{3} t$, then

$$
0 \geq u_{0}^{\prime}(x) \geq-\frac{\epsilon}{2}\left|\frac{u_{0}(x)}{\log \left(u_{0}(x)\right)}\right| \text {. }
$$

We note that $u_{0}$ is a supersolution to eq. (3.25) as a consequence of eq. 3.26). Hence $u_{0}(x) \leq g(x)$ for all $x_{0}-c_{3} t \leq x \leq x_{0}$. Using this, along with the fact that $u_{0}\left(x_{0}\right)=g\left(x_{0}\right)$, we obtain

$$
0 \leq u_{0}\left(x_{0}-c_{3} t\right)-u_{0}\left(x_{0}\right) \leq u_{0}\left(x_{0}-c_{3} t\right)-g\left(x_{0}\right) \leq g\left(x_{0}-c_{3} t\right)-g\left(x_{0}\right) .
$$

To conclude, we estimate the last term, $g\left(x_{0}-c_{3} t\right)-g\left(x_{0}\right)$. Returning to our function $h$ and manipulating the equation, we obtain

$$
\begin{aligned}
g\left(x_{0}-c_{3} t\right)-g\left(x_{0}\right) & =h\left(y_{0}-c_{3} t\right)-h\left(y_{0}\right) \\
& =\exp \left\{-\sqrt{\epsilon\left(y_{0}-c_{3} t\right)}\right\}-\exp \left\{-\sqrt{\epsilon y_{0}}\right\} \\
& =\exp \left\{-\sqrt{\epsilon y_{0}}\right\}\left[\exp \left\{\sqrt{\epsilon y_{0}}\left(1-\sqrt{1-\left(c_{3} t / y_{0}\right)}\right)\right\}-1\right] .
\end{aligned}
$$

It is a simple consequence of Taylor's theorem that there is a constant $C$ such that

$$
1-\sqrt{1-\left(c_{3} t / y_{0}\right)} \leq C\left(c_{3} t / y_{0}\right)
$$

Applying this in the equation above, and using that, by construction, $e^{\sqrt{\epsilon y_{0}}}=\lambda_{t}$, we obtain

$$
g\left(x_{0}-c_{1} t\right)-g\left(x_{0}\right) \leq \lambda_{t}\left[\exp \left\{\frac{C \sqrt{\epsilon} c_{3} t}{\sqrt{y_{0}}}\right\}-1\right]
$$

Then using that $\lambda_{t} \leq c_{1} e^{-c_{2} t}$, we may solve for $y_{0}$ to obtain

$$
y_{0} \geq \frac{1}{\epsilon^{2}}\left(c_{2} t-\log \left(c_{1}\right)\right)^{2} \text {. }
$$


Returning to eq. (3.27), and using that $u_{0}\left(x_{0}\right)=\lambda_{t}$, by construction, we obtain

$$
0 \leq u_{0}\left(x_{0}-c_{3} t\right)-\lambda_{t} \leq g\left(x_{0}-c_{3} t\right)-g\left(x_{0}\right) \leq \lambda_{t}\left[\exp \left\{\epsilon \frac{C c_{3} t}{c_{2} t-\log \left(c_{1}\right)}\right\}-1\right] .
$$

Adding $\lambda_{t}$ to to all sides of each inequality and then dividing by $\lambda_{t}$ yields

$$
1 \leq \frac{u_{0}\left(x_{0}-c_{3} t\right)}{\lambda_{t}} \leq \exp \left\{C^{\prime} \epsilon\right\}
$$

where $C^{\prime}$ is a universal constant which bounds $C c_{3} t /\left(c_{2} t-\log \left(c_{1}\right)\right)$. Again, $C^{\prime}$ can be chosen uniformly in $c_{1}$ as long as $c_{1}$ is bounded above. The result then follows by using the fact that we can choose $\epsilon$ as small as we would like by taking $t$ larger.

We will now prove lemma 3.2 in order to make lemma 3.1 applicable. The idea of the proof is to use the linearized equation to control the growth of $B(m, T)$.

Proof of lemma 3.2. First, we verify that $f_{0}$ is positive. To see this, we simply use the Rayleigh quotient characterization of $f_{0}$ as

$$
f_{0}=\max _{\psi \in H^{1}(\mathbb{T})} \frac{\int\left(f_{u}(x, 0) \psi(x)^{2}-|\nabla \psi(x)|^{2}\right) d x}{\int_{\mathbb{T}} \psi(x)^{2} d x},
$$

where $\mathbb{T}=[0, L]$ with the ends identified. One can verify the positivity of $f_{0}$ by testing the equation with the function $\psi \equiv 1$ and using the positivity of $f_{u}(x, 0)$.

Fix $T>0$, and let $\psi_{0}$ be the unique eigenfunction associated with $f_{0}$ with $\left\|\psi_{0}\right\|_{2}=1$. It follows from the Krein-Rutman theorem that this function exists and is positive [10]. Notice that $\bar{\varphi}(t, x)=\alpha \psi_{0}(x) e^{f_{0} t}$ satisfies

$$
\bar{\varphi}_{t}=\Delta \bar{\varphi}+f_{u}(x, 0) \bar{\varphi} \geq \Delta \bar{\varphi}+f(x, \bar{\varphi}) .
$$

Hence, if $\alpha \psi_{0}(x) \geq B(m, T)$, we have that $\bar{\varphi}(t, x) \geq \varphi^{T, m}(t, x)$ for all $x$ and all $t$, where $\varphi^{T, m}$ is defined by eq. (2.12). Choosing

$$
\alpha=\frac{B(m, T)}{\min _{x} \psi_{0}(x)},
$$

this implies that

$$
B(m, T) \frac{\left\|\psi_{0}\right\|_{\infty}}{\min _{x} \psi_{0}(x)} e^{f_{0} T}=\alpha\left\|\psi_{0}\right\|_{\infty} e^{f_{0} T} \geq f \bar{\varphi}(T, x) d x \geq f \varphi^{T, m}(T, x) d x=m .
$$

Rearranging this inequality yields the lower bound on $B(m, T)$.

The upper bound is similar, but requires slightly more work. We first obtain a bound for small $m$ by using a modulation technique found in [14,15. Notice that our conditions on $f$ guarantee the existence of $M$ such that

$$
f(x, s) \geq f_{u}(x, 0) s-M s^{1+\delta},
$$

holds for all $s \in[0,1]$. Define $\underline{\varphi}(t, x)=\alpha(t) \psi_{0}(x) e^{f_{0} t}$. We find conditions on $\alpha$ to make $\underline{\varphi}$ a sub-solution of $\varphi$. To this end, we compute

$$
\begin{aligned}
\underline{\varphi}_{t}-\underline{\varphi}_{x x}-f(x, \underline{\varphi}) & =\frac{\alpha^{\prime}}{\alpha} \underline{\varphi}+f_{0} \underline{\varphi}-\alpha\left(\psi_{0}\right)_{x x} e^{f_{0} t}-f(x, \underline{\varphi}) \\
& =\frac{\alpha^{\prime}}{\alpha} \underline{\varphi}+f_{0} \underline{\varphi}-\alpha\left[f_{0} \psi_{0}-f_{u}(x, 0) \psi_{0}\right] e^{f_{0} t}-f(x, \underline{\varphi}) \\
& =\frac{\alpha^{\prime}}{\alpha} \underline{\varphi}+f_{0} \underline{\varphi}-f_{0} \underline{\varphi}+f_{u}(x, 0) \underline{\varphi}-f(x, \underline{\varphi}) \\
& \leq \frac{\alpha^{\prime}}{\alpha} \underline{\varphi}+M_{\underline{\varphi}}^{1+\delta}
\end{aligned}
$$


Hence $\underline{\varphi}$ is a sub-solution if we choose

$$
\frac{\alpha^{\prime}}{\alpha^{1+\delta}}=-M e^{f_{0} \delta t}\left\|\psi_{0}\right\|_{\infty}^{\delta} \quad \text { and } \quad \alpha(0)=\frac{B(m, T)}{\left\|\psi_{0}\right\|_{\infty}} .
$$

In other words, we may let

$$
\alpha(t)=\frac{B(m, T)}{\left\|\psi_{0}\right\|_{\infty}}\left[\frac{f_{0} \delta}{B(m, T)^{\delta} M\left[e^{f_{0} \delta t}-1\right]+f_{0} \delta}\right]^{1 / \delta}
$$

Hence at time $t=T$, we may average $\underline{\varphi}$ and use that $\underline{\varphi}$ sits below $\varphi^{T, m}$ to obtain

$$
\frac{B(m, T)}{\left\|\psi_{0}\right\|_{\infty}}\left[\frac{f_{0} \delta}{B(m, T)^{\delta} M\left[e^{f_{0} \delta T}-1\right]+f_{0} \delta}\right]^{1 / \delta} e^{f_{0} T}=f \underline{\varphi}(T, x) d x \leq f \varphi^{T, m}(T, x) d x=m .
$$

Re-arranging this we see that

$$
f_{0} \delta B(m, T)^{\delta} e^{f_{0} \delta T} \leq m^{\delta}\left\|\psi_{0}\right\|_{\infty}^{\delta}\left[B(m, T)^{\delta} e^{f_{0} \delta T} M+f_{0} \delta\right] .
$$

If $m^{\delta} M\left\|\psi_{0}\right\|_{\infty}^{\delta} \leq f_{0} \delta / 2$, then we may rearrange the above to obtain

$$
B(m, T)^{\delta} \leq 2 m^{\delta}\left\|\psi_{0}\right\|_{\infty}^{\delta} e^{-f_{0} \delta T},
$$

finishing the claim for small $m$.

The argument is somewhat more complicated for general $m$. We prove it in two steps. First, we show that there is $R_{1}$, which is uniformly bounded if $m$ is uniformly bounded away from 0 and 1 , such that $B\left(m / 2, T-R_{1}\right) \geq B(m, T)$. To see this, we take $C_{f, m}$ to be a positive constant, arising from parabolic regularity and the smoothness and positivity of $f$, such that $f\left(x, \varphi^{T, m}(t, x)\right) \geq C_{f, m}$ if the average of $\varphi$ is between $m / 2$ and $m$. Then we choose $R_{1}=m /\left(2 C_{f, m}\right)$ and we will show by contradiction that this is the correct choice of $R_{1}$. If not, and instead $B\left(m / 2, T-R_{1}\right)<B(m, T)$ holds, then the maximum principle gives that

$$
\frac{m}{2}=f \varphi^{T-R_{1}, m / 2}\left(T-R_{1}, x\right) d x<f \varphi^{T, m}\left(T-R_{1}, x\right) d x .
$$

Using our choice of $C_{f, m}$ above, we may integrate the equation for $\varphi^{T, m}$ in space for any $t \in\left[T-R_{1}, T\right]$ to obtain

$$
\partial_{t} f \varphi^{T, m}(t, x) d x=f f\left(x, \varphi^{T, m}\right) d x \geq C_{f, m} .
$$

Integrating this in time from $T-R_{1}$ to $T$, using the bound in eq. (3.30), and using our choice of $R_{1}$ yields

$$
m=f \varphi^{T, m}(T, x) d x \geq C_{f, m} R_{1}+f \varphi^{T, m}\left(T-R_{1}, x\right) d x>\frac{m}{2}+\frac{m}{2},
$$

which is clearly a contradiction.

Having proven this fact, we now bootstrap the result for small $m$ to obtain the statement for general $m$. For any $m$, we may find $N$ such that $m / 2^{N}$ is small enough to apply the work above and obtain eq. (3.29). Indeed, we choose $N$ large enough that

$$
\left(\frac{m}{2^{N}}\right)^{\delta} M\left\|\psi_{0}\right\|_{\infty}^{\delta}<f_{0} \delta / 2
$$

and as a result obtain that, for any $\tilde{T}>0$,

$$
B\left(\frac{m}{2^{N}}, \tilde{T}\right) \leq C\left(\frac{m}{2^{N}}\right) e^{-f_{0} \tilde{T}}
$$


In addition to this, we may iterate the procedure above to find $R_{1}, R_{2}, \ldots, R_{N}$ such that

$$
B\left(\frac{m}{2^{i}}, T-\left(R_{1}+\cdots+R_{i}\right)\right) \leq B\left(\frac{m}{2^{i+1}}, T-\left(R_{1}+\cdots+R_{i+1}\right)\right)
$$

holds for any $i$, and hence,

$$
B(m, T) \leq B\left(\frac{m}{2^{N}}, T-\left(R_{1}+\cdots R_{N}\right)\right) .
$$

Combining eqs. (3.31) and 3.32$)$ and defining $C_{m}=C m 2^{-N} e^{-f_{0}\left(R_{1}+\cdots R_{N}\right)}$, we obtain

$$
B(m, T) \leq C \frac{m}{2^{N}} e^{-f_{0}\left(T-R_{1}-R_{2}-\cdots-R_{N}\right)}=C_{m} e^{-f_{0} T},
$$

where we have defined $C_{m}=C m 2^{-N} e^{f_{0}\left(R_{1}+\cdots+R_{N}\right)}$. This concludes the proof.

\section{Characterization of the speed}

In this section, we will show how to characterize $B(m, T)$ in terms of the the global-in-time solution $\varphi$ of eq. (2.11). In the homogeneous case, there is nothing to prove since $\varphi$ and $\varphi^{T, m}$ are equal up to a translation in time, by the uniqueness of solutions of ordinary differential equations. In the inhomogeneous setting, we need to deal with the fact that $\varphi$ is not necessarily flat, while $\varphi^{T, m}(0, x) \equiv B(m, T)$. The idea of the proof is to run the system for time $T / 2$ and use spectral estimates to show that the $\varphi\left(T_{m}-T / 2+t\right)$ and $\varphi^{T, m}(T / 2+t)$ will be close. From then on, they must remain close.

Before we begin, we will need one fact about the global in time solution. We delay the proof of this lemma until section 6 as it will be crucial in the proof of the well-posedness of eq. (2.11).

Lemma 4.1. Let $\varphi$ be any solution of eq. 2.11). Then there exist positive constants $\alpha$ and $\omega$ such that

$$
\lim _{t \rightarrow-\infty} \frac{\varphi(t, x)}{\psi_{0}(x) e^{f_{0} t}}=\alpha, \quad \text { and } \quad \lim _{t \rightarrow \infty} \frac{1-\varphi(t, x)}{\psi_{1}(x) e^{-f_{1} t}}=\omega
$$

where $\psi_{0}$ and $f_{0}$, defined in proposition 2.5, are the normalized eigenfunction and principle eigenvalue of $\Delta+f_{u}(x, 0)$, and where $\psi_{1}$ and $f_{1}$ are the normalized eigenfunction and principle eigenvalue of $\Delta-f_{u}(x, 1)$.

Now, our first step is to prove the following lemma.

Lemma 4.2. Suppose that $\gamma_{0}$ is a $C^{\infty}(\mathbb{T})$ function such that $\left\|\gamma_{0}\right\|_{\infty} \leq C e^{-f_{0} T}$ for some $C>0$.

$$
\left\{\begin{array}{l}
\gamma_{t}=\gamma_{x x}+f(x, \gamma), \\
\gamma(0, x)=\gamma_{0}(x) .
\end{array}\right.
$$

Fix $\epsilon>0$. Then there is $T_{0}>0$, which can be chosen uniformly if $C$ is bounded above, such that if $T \geq T_{0}$, we have that

$$
\left\|\gamma(T / 2)-\psi_{0}\left(\int \gamma_{0} \psi_{0}\right) e^{f_{0} T / 2}\right\|_{2} \leq \epsilon e^{-f_{0} T / 2} .
$$

Proof. Define

$$
\Gamma(t, x)=\gamma(t, x) e^{f_{0}(T-t)}
$$

and we can easily see that $\Gamma$ satisfies

$$
\Gamma_{t}-\Gamma_{x x}+f_{0} \Gamma=f(x, \gamma) e^{f_{0}(T-t)}=\left(\frac{f(x, \gamma)}{\gamma}\right) \Gamma .
$$


Letting $\Gamma_{1}(t)=\int \Gamma(t, x) \psi_{0}(x) d x$, we notice that

$$
\dot{\Gamma}_{1}=e^{f_{0}(T-t)} \int\left(f(x, \gamma)-f_{u}(x, 0) \gamma\right) \psi_{0}(x) d x .
$$

First, we notice that $\dot{\Gamma}_{1} \leq 0$. In addition, since $\psi_{0}$ multiplied by a large constant is a super-solution of $\Gamma$, it follows that $\Gamma$ is uniformly bounded. We utilize this, along with eq. (3.28), to obtain

$$
\begin{aligned}
0 \geq \dot{\Gamma}_{1} & \geq-M e^{f_{0}(T-t)} \int \gamma(x)^{1+\delta} \psi_{0}(x) d x \geq-M C^{\prime} e^{f_{0}(T-t)} e^{\delta f_{0}(t-T)} \int \gamma(x) \psi_{0}(x) d x \\
& \geq-M C^{\prime} e^{\delta f_{0}(t-T)} \Gamma_{1} .
\end{aligned}
$$

Dividing by $\Gamma_{1}$, integrating in time, and exponentiating yields

$$
1 \geq \frac{\Gamma_{1}(T / 2)}{\Gamma_{1}(0)} \geq \exp \left\{-e^{-\frac{\delta f_{0}}{2} T} C^{\prime \prime}\left[1-e^{-\frac{\delta f_{0}}{2} T}\right]\right\} .
$$

Defining $\gamma_{1}(t)=\int \gamma(t, x) \psi_{0}(x) d x$, we may rewrite this inequality as

$$
\left|\gamma_{1}(T / 2)-e^{f_{0} T / 2} \gamma_{1}(0)\right| \leq o(1) e^{-f_{0} T / 2}
$$

Now we let $\Gamma_{2}(t, x)=\Gamma(t, x)-\Gamma_{1}(t) \psi_{0}(x)$, and we bound $\Gamma_{2}$. Notice that $\Gamma_{2}$ satisfies the equation

$$
\begin{aligned}
\left(\Gamma_{2}\right)_{t}- & \left(\Gamma_{2}\right)_{x x}-\left(f_{u}(x, 0)-f_{0}\right) \Gamma_{2} \\
& =\left[f(x, \gamma) e^{f_{0}(T-t)}-f_{u}(x, 0) \Gamma\right]-\psi_{0}(x) e^{f_{0}(T-t)} \int\left(f(y, \gamma)-f_{u}(y, 0) \gamma(y)\right) \psi_{0}(y) d y
\end{aligned}
$$

Because $\Gamma_{2}$ is orthogonal to the the eigenspace for eigenvalue $f_{0}$ of $\Delta+f_{u}(x, 0)$, then there is $\alpha$ such that

$$
\int\left[\left(\Gamma_{2}\right)_{x x}+\left(f_{u}(x, 0)-f_{0}\right) \Gamma_{2}\right] \Gamma_{2} d x \leq-\alpha\left\|\Gamma_{2}\right\|_{2}^{2} .
$$

Hence, multiplying eq. (4.34) by $\Gamma_{2}$ and, again, using that $\Gamma_{2}$ and $\psi_{0}$ are perpendicular yields

$$
\begin{aligned}
\frac{1}{2} \frac{d}{d t}\left\|\Gamma_{2}\right\|_{2}^{2}+\alpha\left\|\Gamma_{2}\right\|_{2}^{2} & \leq e^{f_{0}(T-t)} \int\left[f(x, \gamma)-f_{u}(x, 0) \gamma\right] \Gamma_{2} d x \\
& \leq M e^{f_{0}(T-t)} \int \gamma^{1+\delta}\left|\Gamma_{2}\right| d x=M e^{\delta f_{0}(t-T)} \int \Gamma^{1+\delta} \Gamma_{2} d x
\end{aligned}
$$

As we noted above, $\|\Gamma\|_{\infty}$ is uniformly bounded in time. Hence, we may write this as

$$
\frac{d}{d t}\left\|\Gamma_{2}\right\|_{2}^{2}+2 \alpha\left\|\Gamma_{2}\right\|_{2}^{2} \leq C e^{\delta f_{0}(t-T)}\left\|\Gamma_{2}\right\|_{2},
$$

where $C$ is a new constant independent of $t$ and $T$. Solving this differential inequality and using that $\left\|\Gamma_{2}(0)\right\| \leq C$ for some constant $C$, yields constants $C>0$ and $\theta>0$ such that $\left\|\Gamma_{2}(T / 2)\right\|_{2} \leq C e^{-\theta T}$.

The combination of this inequality with eq. (4.33) yields that

$$
\left\|\gamma(T / 2)-e^{f_{0} T / 2} \gamma_{1}(0) \psi_{0}\right\|_{2} \leq C\left|\gamma_{1}(T / 2)-e^{f_{0} T / 2} \gamma_{1}(0)\right|+e^{-f_{0} T / 2}\left\|\Gamma_{2}\right\|_{2} \leq o(1) e^{-f_{0} T / 2},
$$

finishing the proof.

We now show how to use this lemma to conclude proposition 2.5. Fix $m \in(0,1)$ and define the starting time $S(m, T)$ as the time such that

$$
\int \varphi(S(m, T), x) \psi_{0}(x) d x=B(m, T) \int \psi_{0} .
$$


We first show that $f \varphi(S(m, T)+T, x) d x \rightarrow m$ as $T$ tends to infinity. Then, defining $T_{m}$ as the unique time such that

$$
f \varphi\left(T_{m}, x\right) d x=m
$$

we will leverage this to show that

$$
\lim _{T \rightarrow \infty} \frac{\int \varphi\left(T_{m}-T, x\right) \psi_{0}(x) d x}{B(m, T) \int \psi_{0}}=1
$$

finishing the proof.

By parabolic regularity, lemma 4.2, and lemma 3.2, we have that

$$
\left\|\varphi(S(m, T)+T / 2)-\varphi^{T, m}(T / 2)\right\|_{2} \leq o(1) e^{-f_{0} T / 2},
$$

where $o(1)$ tends to zero as $T$ tends to infinity (uniformly for $m$ bounded away from 1). By parabolic regularity this may be strengthened to

$$
\left\|\varphi(S(m, T)+T / 2)-\varphi^{T, m}(T / 2)\right\|_{\infty} \leq o(1) e^{-f_{0} T / 2} .
$$

Define $\eta(t, x)=\varphi(S(m, T)+T / 2+t, x)-\varphi^{T, m}(T / 2+t, x)$ and notice that $\eta$ solves

$$
\eta_{t}-\eta_{x x}=c(t, x) \eta
$$

where $c(t, x)=\left(f\left(x, \varphi^{1}\right)-f\left(x, \varphi^{2}\right)\right) /\left(\varphi^{1}-\varphi^{2}\right) \leq f_{u}(x, 0)$. Hence, $o(1) \psi_{0} e^{f_{0}(t-T / 2)}$ is a super-solution to the equation above with initial data larger than $\eta(0, x)$. This implies that

$$
|\eta(T / 2, x)| \leq o(1) \psi(x) \leq o(1)
$$

holds for every $x$. Using the definition of $\eta$, this implies that

$$
\left\|\varphi(S(m, T)+T, \cdot)-\varphi^{T, m}(T, \cdot)\right\|_{\infty} \leq o(1) .
$$

By our definition of $\varphi^{T, m}$, this implies that

$$
\lim _{T \rightarrow \infty} f \varphi(S(m, T)+T, x) d x=m,
$$

holds, where the limit may be taken uniformly in $m$ if $m$ is bounded away from 1 .

Now we will show eq. (4.36). Suppose this does not hold. Let $\theta>1$, without loss of generality, be such that there is a sequence $T_{1}, T_{2}, T_{3}, \ldots$ such that

$$
\lim _{n \rightarrow \infty} \frac{\int \varphi\left(T_{m}-T_{n}, x\right) \psi_{0}(x) d x}{B\left(m, T_{n}\right) \int \psi_{0}}>\theta .
$$

We note that the proof is similar for $\theta<1$ and the limit above is less than $\theta$. Using lemma 4.1 and the definition of $S(m, T)$, we can choose $N_{0}$ be such that if $n \geq N_{0}$ then

$$
\frac{e^{T_{m}-T_{n}}}{e^{S\left(m, T_{n}\right)}}>\theta
$$

and hence,

$$
T_{m}-T_{n}-S\left(m, T_{n}\right)>\log (\theta)>0 .
$$

By parabolic regularity, along with the smoothness and positivity of $f$, we may find $\epsilon$ depending only on $m$ and $\theta$ such that $f(x, \varphi(t, x))>\epsilon$ for all $t \in\left[T_{n}+S\left(m, T_{n}\right), T_{m}\right]$. This holds because $\varphi$ is bounded away from 0 and 1 on this interval. Integrating the equation for $\varphi$ on this time interval yields

$$
\varphi\left(T_{m}\right)-\varphi\left(T_{n}+S\left(m, T_{n}\right)\right)=\int_{T_{n}+S\left(m, T_{n}\right)}^{T_{m}} f(x, \varphi) d x \geq \epsilon \log (\theta) .
$$

The average of the first term is $m$, and the average of the second term converges to $m$ for large $n$. This implies that the left hand side tends to zero, which is a contradiction. This finishes the proof. 


\section{Convergence to the global in time problem}

In this section, we prove theorem 2.7. We will do this in two steps. First we will prove the closeness of $u$ and $\varphi^{T, m}$. Then we will show that $\varphi^{T, m}$ and $\varphi$ are close when suitably translated in time. To this end, we state two lemmas.

Lemma 5.1. Define $\varphi^{T, m}$ as in eq. (2.12), and define $I_{m, T}$ to be the interval $[n L, n L+L)$ containing $u_{0}^{-1}(B(m, T))$, we have that

$$
\lim _{T \rightarrow \infty} \max _{m \in(0,1)}\left\|u(T, \cdot)-\varphi^{T, m}(T, \cdot)\right\|_{L^{\infty}\left(I_{m, T}\right)}=0 .
$$

Lemma 5.2. Define $S(m, T)$ as in eq. (4.35). Then,

$$
\lim _{T \rightarrow \infty} \max _{t \in[T / 2, \infty]}\left\|\varphi(S(m, T)+t, \cdot)-\varphi^{T, m}(t, \cdot)\right\|_{\infty}=0 .
$$

This limit can be taken uniformly for $m$ which is bounded away from 1.

We will prove eq. (5.37) at the end of this section. The proof of lemma 5.2 follows from our choice of $S(m, T)$ and from work in lemma 4.2. Hence, we omit it. We now show how to conclude theorem 2.7 using these two lemmas.

Proof of theorem 2.7. First, denote $I_{m, T}$ as $[n L, n L+L]$. Define $T^{S_{n}}$ as the unique time such that

$$
f \varphi\left(T^{S_{n}}, x\right) d x=S_{n}
$$

where $S_{n}$ is as in eq. 2.15). We show that if $T$ (or equivalently $n$ ) is large enough, then $T^{S_{n}}$ and $S(m, T$ ) are close, and that we can obtain this in a uniform way for $m$ bounded away from 1 . To this end, notice that, by the definition of $I_{m, T}$,

$$
u_{0}(n L) \geq B(m, T) \geq u_{0}(n L+L) .
$$

In addition, by lemma 3.1 and lemma 3.2 , we have that

$$
\frac{u_{0}(n L)}{B(m, T)}=1+o(1)
$$

where $o(1)$ tends to zero as $T$ tends to infinity in a uniform way if $m$ is bounded away from 1 . Using lemma 4.1, we then compute that

$$
\begin{aligned}
1+o(1) & =\frac{u_{0}(n L)}{B(m, T)}=\left(\frac{f \varphi\left(T^{S_{n}}, x\right) d x}{\left(\int \psi_{0}(x) d x\right)^{2}}\right)\left(\frac{\int \psi_{0} d x}{\int \varphi(S(m, T), x) \psi_{0}(x) d x}\right) \\
& =\left(\frac{f \alpha\left(\psi_{0}(x)+o(1)\right) e^{f_{0} T^{S_{n}}} d x}{\left(\int \psi_{0}(x) d x\right)^{2}}\right)\left(\frac{\int \psi_{0} d x}{\int \alpha\left(\psi_{0}(x)+o(1)\right) e^{f_{0} S(m, T)} \psi_{0}(x) d x}\right)=\frac{e^{f_{0} T^{S_{n}}}}{e^{f_{0} S(m, T)}}(1+o(1)) .
\end{aligned}
$$

Again, the constant $o(1)$ tends to zero uniformly as long as $m$ is bounded away from 1 . This yields the claim that $T^{S_{n}}-S(m, T)$ tends to zero as $T$ tends to infinity.

Fix $\epsilon>0$. Using lemma 5.1, choose $T_{1}$ large enough that if $T \geq T_{1}$, then

$$
\max _{m \in(0,1)}\left\|u(T, \cdot)-\varphi^{T, m}(T, \cdot)\right\|_{L^{\infty}\left(I_{m, T}\right)} \leq \epsilon / 2
$$

In addition, choose $m_{1}<1$, depending only on $f$ and $\epsilon$, such that if $m \geq m_{1}$, then

$$
\varphi^{T, m}(T, x) \geq 1-\epsilon / 2,
$$


for all $x \in \mathbb{T}$ and any $T$.

We will consider three cases. First, if $n$ is small enough that $\varphi\left(T^{S_{n}}, x\right) \geq B\left(m_{1}, T\right)$, the maximum principle implies that

$$
\varphi\left(T^{S_{n}}+T, x\right) \geq \varphi^{T, m_{1}}(T, x) \geq 1-\epsilon / 2 .
$$

This, combined with eq. (5.39), implies that

$$
\left\|\varphi\left(T^{S_{n}}+T, x\right)-u(T, \cdot)\right\|_{L^{\infty}([n L, n L+L])} \leq \epsilon,
$$

for $T \geq T_{1}$.

The second case is when $n$ is such that $f \varphi\left(T^{S_{n}}, x\right) d x \leq B\left(m_{1}, T\right)\left(\int \psi_{0}\right)^{2}$. This implies that $m \leq m_{1}$. Indeed, using the definition of $S_{n}$, we obtain that

$$
B\left(m_{1}, T\right)\left(\int \psi_{0}\right)^{2} \geq f \varphi\left(T^{S_{n}}, x\right) d x=S_{n}=u_{0}(n L)\left(\int \psi_{0}\right)^{2} \geq B(m, T)\left(\int \psi_{0}\right)^{2} .
$$

Thus, $B(m, T) \leq B\left(m_{1}, T\right)$, implying that $m \leq m_{1}$. Hence we may find $T_{2}$, depending only on $m_{1}, f$, and $\epsilon$, such that if $T \geq T_{2}$ then $T^{S_{n}}$ is close enough to $S(m, T)$ that

$$
\left\|\varphi\left(T^{S_{n}}+T, \cdot\right)-\varphi(S(m, T)+T, \cdot)\right\|_{L^{\infty}} \leq \epsilon / 8 .
$$

In addition, increasing $T_{2}$ if necessary, we may apply lemma 5.2 to get that if $T \geq T_{2}$ then

$$
\left\|\varphi(S(m, T)+T, \cdot)-\varphi^{T, m}(T, \cdot)\right\|_{\infty} \leq \epsilon / 16 .
$$

The combination of these two inequalities with eq. (5.39) implies that if $T \geq T_{1}+T_{2}$, then

$$
\left\|\varphi\left(T^{S_{n}}+T, \cdot\right)-u(T, \cdot)\right\|_{L^{\infty}([n L, n L+L])} \leq \epsilon .
$$

Finally, we handle the case when neither of these is true. That both of these conditions do not hold, along with parabolic regularity, implies that we may find a positive constant $\theta<1$, which depends only on $m_{1}$ and $f$, such that

$$
\theta f \varphi\left(T^{S_{n}}, x\right) d x \leq B\left(m_{1}, T\right)\left(\int \psi_{0}\right)^{2} .
$$

Using lemma 3.2 we may find $t_{0}>0$, depending only on $f$ and $m_{1}$, such that

$$
f \varphi\left(T^{S_{n}}, x\right) d x \leq B\left(m_{1}, T-t_{0}\right)\left(\int \psi_{0}\right)^{2} .
$$

Following the work above, we then have that $B(m, T) \leq B\left(m_{1}, T-t_{0}\right)$. Hence we obtain

$$
(1-m)=f\left(1-\varphi^{T, m}(T, x)\right) d x \geq f\left(1-\varphi^{T-t_{0}, m_{1}}(T, x)\right) d x \geq C e^{f_{1} t_{0}} .
$$

The constant $C$ depends only on $m_{1}$ and $f$, and the last inequality comes from considering a constant multiple of $\psi_{1} e^{f_{1} t}$ as a sub-solution to $1-\varphi^{T-t_{0}}$ on the interval $\left[T-t_{0}, T\right]$. This shows that $m$ is bounded away from 1 independent of $T$. Hence we may argue exactly as in the last paragraph, finishing the proof.

We will now show how to conclude lemma 5.1. The main idea is largely the same: when $m$ is large enough, both $u$ and $\varphi^{T, m}$ are near 1 , and when $m$ is bounded from 1, we may take the limit in $T$ uniformly in $m$. 
Proof of lemma 5.1. Fix $\epsilon>0$, and we will choose $T$, independent of $m$, such that

$$
\left\|u(T)-\varphi^{T, m}(T)\right\|_{L^{\infty}\left(I_{m, T}\right)} \leq \epsilon .
$$

To this end, we utilize the work from section 3 .

First, we may use parabolic regularity to find $m_{1}<1$ depending only on $f$ and $\epsilon$ such that $1-\varphi^{T, m_{1}}(T, x) \leq \epsilon / 2$. We may now consider, separately, the cases when $m \leq m_{1}$ and when $m \geq m_{1}$.

If $m \geq m_{1}$, using a sub-solution, $v=\varphi^{T, m_{1}} \underline{w}$, as in section 3.2 , we may choose $T$, depending only on $\epsilon$ and $f$, such that

$$
\underline{w}(T, x) \geq(1-\epsilon) /(1-\epsilon / 2),
$$

for any $x \leq \max I_{m_{1}, T}$. Hence, we have that

$$
1 \geq u(T, x) \geq v(T, x) \geq \varphi^{T, m_{1}}(T, x) w_{\ell} \geq(1-\epsilon / 2) \frac{1-\epsilon}{1-\epsilon / 2}=1-\epsilon .
$$

for any $x \leq \max I_{m_{1}, T}$. By the maximum principle, we can see that $\varphi^{T, m} \geq \varphi^{T, m_{1}}$ if $m \geq m_{1}$. Hence we have that, if $m \geq m_{1}$

$$
\left\|u(T)-\varphi^{T, m}\right\|_{L^{\infty}\left(I_{m, T}\right)} \leq \epsilon .
$$

The case when $m \leq m_{1}$ follows from our work above. Indeed, since $m$ is bounded away from 1, we may choose $T$ such that $\bar{w}$ and $\underline{w}$ from section 3.1 and section 3.2 , respectively, satisfy

$$
1-\epsilon / 2 \leq \underline{w}(T, x), \quad \text { and } \quad \bar{w}(T, x) \leq 1+\epsilon / 2,
$$

for any $x \in I_{m, T}$. Hence we have that, for any $x \in I_{m, T}$,

$$
\left(1-\frac{\epsilon}{2}\right) \varphi^{T, m}(T, x) \leq \underline{w}(T, x) \varphi^{T, m}(T, x) \leq u(T, x) \leq \bar{w}(T, x) \varphi^{T, m}(T, x) \leq\left(1+\frac{\epsilon}{2}\right) \varphi^{T, m}(T, x),
$$

which finishes the proof.

\section{Well-posedness of the global in time solution}

In this section, we establish the existence and uniqueness (up to translation) of the solution to eq. (2.11). In other words, we prove the following proposition.

Proposition 6.1. There exists a unique global in time solution of eq. (2.11).

We will prove this in a series of three lemmas. First, we will exhibit the existence of a solution. Afterwards, we show that any solution to eq. (2.11) must look grow and decay exponentially as $t$ tends to $-\infty$ and $\infty$, respectively. Finally, we will use this qualitative property and the sliding method to establish uniqueness.

Lemma 6.2. There exists a solution of eq. (2.11).

Proof. We establish existence by considering the limit of family of initial value problems. Define $\varphi^{n}$ to be the solution to the parabolic equation on $\left[T_{n}, \infty\right] \times \mathbb{T}$

$$
\left\{\begin{array}{l}
\varphi_{t}^{n}=\varphi_{x x}^{n}+f\left(x, \varphi^{n}\right) \\
f \varphi^{n}(0, x) d x=1 / 2 \\
\varphi^{n}\left(T_{n}, x\right) \equiv 1 / n
\end{array}\right.
$$

where $T_{n} \leq 0$ is an unknown. It is easy to check that $\left(n \min \psi_{0}\right)^{-1} \psi_{0}(x) e^{f_{0}\left(t-T_{n}\right)}$ is a super-solution, where $\psi_{0}$ and $f_{0}$ are the principle eigenfunction and eigenvalue of $\Delta+f_{u}(x, 0)$. Thus, we obtain

$$
\frac{1}{2}=f \varphi^{n}(0, x) d x \leq \frac{\left\|\psi_{0}\right\|_{\infty}}{n \min \psi_{0}} e^{-f_{0} T_{n}} .
$$


This implies that $T_{n} \leq C \log (n)$, for some constant $C$. Hence we may use parabolic regularity, along with the compactness of Hölder spaces to obtain convergence along a subsequence to a global in time function $\varphi$ which satisfies

$$
\left\{\begin{array}{l}
\varphi_{t}=\varphi_{x x}+f(x, \varphi), \\
f \varphi(0, x) d x=1 / 2
\end{array}\right.
$$

finishing the proof.

Now we establish that the asymptotics of any solution $\varphi$ to eq. $(2.11)$ look like the solutions to the linearized problems around 0 and 1 . This amounts to proving lemma 4.1, as stated in section 4.

Proof of lemma 4.1. We will prove the claim for $t$ tending to $-\infty$. The result for $t$ tending to infinity follows by looking at $\tilde{\varphi}(t, x)=1-\varphi(-t, x)$ and arguing similarly. Our strategy is to first show that $\varphi$ is bounded above and below by a multiple of $e^{f_{0} t}$. Then we will appeal to parabolic regularity to obtain the result.

To this end, we first assume, by contradiction, that $e^{-f_{0} t} \varphi(t, x)$ tends to zero along some sequence $t_{n}$, tending to $-\infty$, for some choice of $x_{n}$. By the parabolic Harnack inequality, it must converge to zero uniformly in space. Hence, we may assume that, for any $\epsilon$, there exists $N$ such that if $n \geq N$ then $\left\|e^{-f_{0} t_{n}} \varphi\left(t_{n}\right)\right\|_{\infty} \leq \epsilon$. In this case, we may apply lemma 3.2 to find $t_{n}$ large enough that $\varphi^{-t_{n}, 1 / 4}\left(t-t_{n}, x\right)$ is a super-solution to $\varphi$ on $\left[-t_{n}, 0\right] \times \mathbb{T}$. Using this inequality at time $t=0$, we have that

$$
\frac{1}{4}=f \varphi^{-t_{n}, 1 / 4}\left(-t_{n}, x\right) d x \geq f \varphi(0, x) d x=\frac{1}{2} .
$$

This is clearly a contradiction, finishing the lower bound.

Now we will show that there is some $C$ such that $e^{-f_{0} t} \varphi(t, x) \leq C$ for $t \leq 0$. To this end, suppose that there is some sequence $t_{n}$ tending to $-\infty$ such that $e^{-f_{0} t_{n}} \varphi\left(t_{n}, x_{n}\right) \rightarrow \infty$ for some sequence $x_{n}$. Again, by the parabolic Harnack inequality, this implies that $\min e^{-f_{0} t_{n}} \varphi\left(t_{n}\right) \rightarrow \infty$. Hence, we may apply lemma 3.2 and take $n$ large enough that $\varphi^{-t_{n}, 3 / 4}\left(t-t_{n}, x\right)$ is a sub-solution of $\varphi$ on $\left[-t_{n}, 0\right] \times \mathbb{T}$. Using this inequality at time $t=0$ yields

$$
\frac{3}{4}=f \varphi^{-t_{n}, 3 / 4}\left(-t_{n}, x\right) d x \leq f \varphi(0, x) d x=\frac{1}{2} .
$$

This is a contradiction, giving us the desired upper bound.

Now, letting $\Phi(t, x)=e^{-f_{0} t} \varphi(t, x)$, we will show that $\Phi$ converges to $\alpha \psi_{0}$. To this end, we may write $\Phi_{1}=\int \Phi(t, x) \psi_{0}(x) d x$ and $\Phi_{2}=\Phi-\psi_{0} \Phi_{1}$. Then as in our work in lemma 4.2, we may see that there are positive constants $C$ and $\theta$, independent of time such that

$$
\begin{aligned}
& 0 \geq \dot{\Phi}_{1} \geq-C e^{\delta f_{0} t} \Phi_{1}, \\
& \frac{d}{d t}\left\|\Phi_{2}\right\|_{2}^{2}+2 \theta\left\|\Phi_{2}\right\|_{2}^{2} \leq C e^{\delta f_{0} t}\left\|\Phi_{2}\right\|_{2} .
\end{aligned}
$$

Solving these differential inequalities and using that $\Phi$ is bounded for all time gives us that, as $t$ tends to $-\infty, \Phi_{1}(t) \rightarrow \alpha$, for some $\alpha$, and that $\Phi_{2}(t) \rightarrow 0$. Since $\Phi$ is bounded away from zero, we get that $\alpha>0$. This gives us the desired result.

Finally, we use this qualitative information, along with the sliding method, to prove uniqueness of any solution to eq. (2.11).

Lemma 6.3. Any solution to eq. (2.11) is unique.

Proof. Suppose that $\varphi^{1}$ and $\varphi^{2}$ are both solutions to eq. (2.11) with $f \varphi^{i}(0, x) d x=1 / 2$. Using lemma 4.1, we may choose $h$ large enough such that

$$
\varphi^{1}(t, x) \leq \varphi^{2}(t+h, x)
$$


holds for all $t$ and all $x$. Then we define

$$
h_{0}=\inf \left\{h: \varphi^{1}(t, x)<\varphi^{2}(t+h, x)\right\} .
$$

Since $\varphi^{1}$ and $\varphi^{2}$ have the same spatial average at $t=0$, then $h_{0} \geq 0$. We wish to show that $h_{0}=0$. To this end, let us assume that $h_{0}>0$. There are two cases. Using lemma 3.2, we can find $\alpha_{1}$ and $\alpha_{2}$ such that $\varphi^{1}(t, x) \rightarrow \alpha_{1} \psi_{0}$ as $t$ tends to $-\infty$ that $\varphi^{2}(t, x) \rightarrow \alpha_{2} \psi_{0}$ as $t$ tends to $-\infty$. The first case is that $\alpha_{1}=e^{f_{0} h_{0}} \alpha_{2}$. In other words, $\varphi^{1}$ and $\varphi^{2}$ touch at time $t=-\infty$. Define $\eta(t, x)=\varphi^{2}\left(t+h_{0}, x\right)-\varphi^{1}(t, x)$ and $\eta$ satisfies

$$
\eta_{t}=\eta_{x x}+\frac{f\left(x, \varphi^{2}\left(t+h_{0}, x\right)\right)-f\left(x, \varphi^{1}(t, x)\right)}{\varphi^{2}\left(t+h_{0}, x\right)-\varphi^{1}(t+h, x)} \eta
$$

Fix $T<0$ to be determined later. Since $\varphi^{i} \sim \alpha_{i} e^{f_{0} T}$ and since $\alpha_{1}=e^{f_{0} h_{0}} \alpha_{2}$, we notice that $\eta(T, x) \leq$ $o(1) e^{f_{0} T}$ where $o(1)$ tends to zero as $T$ tends to $-\infty$. From this it is easy to see that $o(1) \psi_{0} e^{f_{0} t}$ is a supersolution to equation for $\eta$ starting from any time $T$. This implies that $\eta(0, x) \leq o(1)$. Since this holds independent of our choice of $T$, we get that that $\eta(0, x)=0$.

On the other hand, $\varphi^{2}$ is bounded away from 0 and 1 on the time interval $\left[0, h_{0}\right]$. Hence, there exists $\theta>0$ such that $f\left(x, \varphi^{2}\right) \geq \theta$ on the time interval $\left[0, h_{0}\right]$. Integrating the equation for $\varphi^{2}$ in time and space, along with this fact and the fact that $\eta(0, x)=0$, yields

$$
\begin{aligned}
0 & =f \eta(0, x) d x=f \varphi^{2}\left(h_{0}, x\right) d x-f \varphi^{1}(0, x) d x=f \varphi^{2}\left(h_{0}, x\right) d x-m \\
& =f \varphi^{2}\left(h_{0}, x\right) d x-f \varphi^{2}(0, x) d x=\int_{0}^{h_{0}} f f\left(x, \varphi^{2}(s, x)\right) d x d s \geq \int_{0}^{h_{0}} \theta d s=h_{0} \theta>0 .
\end{aligned}
$$

The second equality on the second line comes from integrating the equation for $\varphi^{2}$ in time and space. The above inequality is clearly a contradiction.

Hence, it must be that, instead, $\alpha_{1}<e^{f_{0} h_{0}} \alpha_{2}$. Choose $\epsilon>0$ small enough that $\alpha_{1} e^{\epsilon}<e^{h_{0}-2 \epsilon} \alpha_{2}$. Take $t_{0}$ to be a very large negative number such that if $t \leq t_{0}$ then

$$
\varphi^{1}(t, x) \leq \alpha_{1} e^{\epsilon} \psi_{0}(x) e^{f_{0} t}
$$

and

$$
\varphi^{2}\left(t+h_{0}\right) \geq \alpha_{2} e^{f_{0}\left(t+h_{0}-\epsilon\right)} \psi_{0}(x)
$$

Notice then that $\varphi^{2}\left(t+h_{0}-\epsilon, x\right)>\varphi^{1}(t, x)$ for all $t<t_{0}$. In addition, the comparison principle assures us that $\varphi^{2}\left(t+h_{0}-\epsilon, x\right)>\varphi^{1}(t, x)$ for all $t \geq t_{0}$ as well. This contradicts our choice of $h_{0}$ in eq. (6.40).

Hence we may conclude that $h_{0}$ must be zero. This implies that $\varphi^{1}(t, x) \leq \varphi^{2}(t, x)$ for all $t$ and all $x$. The proof may be applied just as easily to show that $\varphi^{2} \leq \varphi^{1}$ as well. Hence we conclude that $\varphi^{1} \equiv \varphi^{2}$, concluding the proof.

\section{Acknowledgements}

The author wishes to thank Jun Gao, Yu Gu, and Lenya Ryzhik for many helpful discussions.

\section{References}

[1] R. F. Bass, Diffusions and elliptic operators, Probability and its Applications (New York), SpringerVerlag, New York, 1998.

[2] H. Berestycki and L. Nirenberg, Travelling fronts in cylinders, Ann. Inst. H. Poincaré Anal. Non Linéaire 9 (1992), no. 5, 497-572. 
[3] E. Bouin, V. Calvez, N. Meunier, S. Mirrahimi, B. Perthame, G. Raoul, and R. Voituriez, Invasion fronts with variable motility: phenotype selection, spatial sorting and wave acceleration, C. R. Math. Acad. Sci. Paris 350 (2012), no. 15-16, 761-766.

[4] E. Bouin, V. Calvez, and G. Nadin, Propagation in a kinetic reaction-transport equation: travelling waves and accelerating fronts, preprint (2014), http://arxiv.org/abs/1307.8325.

[5] M. Bramson, Maximal displacement of branching Brownian motion, Comm. Pure Appl. Math. 31 (1978), no. $5,531-581$.

[6] Convergence of solutions of the Kolmogorov equation to travelling waves, Mem. Amer. Math. Soc. 44 (1983), no. 285, iv+190.

[7] X. Cabré, A.-C. Coulon, and J.-M. Roquejoffre, Propagation in Fisher-KPP type equations with fractional diffusion in periodic media, C. R. Math. Acad. Sci. Paris 350 (2012), no. 19-20, 885-890.

[8] X. Cabré and J.-M. Roquejoffre, The influence of fractional diffusion in Fisher-KPP equations, Comm. Math. Phys. 320 (2013), no. 3, 679-722.

[9] A.-C. Coulon and J.-M. Roquejoffre, Transition between linear and exponential propagation in FisherKPP type reaction-diffusion equations, Comm. Partial Differential Equations 37 (2012), no. 11, 2029 2049.

[10] R. Dautray and J.-L. Lions, Mathematical analysis and numerical methods for science and technology. Vol. 3, Springer-Verlag, Berlin, 1990, Spectral theory and applications, With the collaboration of Michel Artola and Michel Cessenat, Translated from the French by John C. Amson.

[11] R. Fisher, The wave of advance of advantageous genes, Ann. Eugenics 7 (1937), 355-369.

[12] J. Garnier, Accelerating solutions in integro-differential equations, SIAM J. Math. Anal. 43 (2011), no. 4, 1955-1974.

[13] J. Gärtner, Location of wave fronts for the multidimensional KPP equation and Brownian first exit densities, Math. Nachr. 105 (1982), 317-351.

[14] F. Hamel, J. Nolen, J.-M. Roquejoffre, and L. Ryzhik, The logarithmic delay of KPP fronts in a periodic medium, preprint (2012), http://arxiv.org/abs/1211.6173.

[15] _ A short proof of the logarithmic Bramson correction in Fisher-KPP equations, Netw. Heterog. Media 8 (2013), no. 1, 275-289.

[16] F. Hamel and L. Roques, Fast propagation for KPP equations with slowly decaying initial conditions, J. Differential Equations 249 (2010), no. 7, 1726-1745.

[17] C. Henderson, Population stabilization in branching Brownian motion with absorption and drift, Commun. Math. Sci. (to appear), http://arxiv.org/abs/1409.4836.

[18] A.N. Kolmogorov, I.G. Petrovskii, and N.S. Piskunov, Étude de l'équation de la chaleurde matière et son application à un problème biologique, Bull. Moskov. Gos. Univ. Mat. Mekh. 1 (1937), 1-25, See 24] pp. 105-130 for an English translation.

[19] M. Kot, M. Lewis, and P. Van den Driessche, Dispersal data and the spread of invading organisms, Ecology 77 (1996), 2027-2042.

[20] N. V. Krylov, Lectures on elliptic and parabolic equations in Hölder spaces, Graduate Studies in Mathematics, vol. 12, American Mathematical Society, Providence, RI, 1996. 
[21] Lectures on elliptic and parabolic equations in Sobolev spaces, Graduate Studies in Mathematics, vol. 96, American Mathematical Society, Providence, RI, 2008.

[22] K.-S. Lau, On the nonlinear diffusion equation of Kolmogorov, Petrovsky, and Piscounov, J. Differential Equations 59 (1985), no. 1, 44-70.

[23] J. Medlock and M. Kot, Spreading disease: Integro-differential equations old and new, Mathematical Biosciences 184 (2003), 201-222.

[24] P. Pelcé (ed.), Dynamics of curved fronts, Perspectives in Physics, Academic Press Inc., Boston, MA, 1988.

[25] M. I. Roberts, A simple path to asymptotics for the frontier of a branching Brownian motion, Ann. Probab. 41 (2013), no. 5, 3518-3541.

[26] K. Uchiyama, The behavior of solutions of some nonlinear diffusion equations for large time, J. Math. Kyoto Univ. 18 (1978), no. 3, 453-508. 